\title{
Design and fabrication of a compact, high-performance interference-filter-based external-cavity diode laser for use in the China Space Station
}

\author{
Lingqiang Meng (孟令强) $)^{1,2}$, Pengyang Zhao (赵范杨) ${ }^{1,2}$, Fanchao Meng (孟范超) ${ }^{1}$, Long Chen (陈 龙) \\ Yong Xie (谢 永) ${ }^{2}$, Yikun Wang (王义坤 $)^{1,2}$ ，Wei Bian (边 伟) ${ }^{1}$ ， Jianjun Jia (贾建军) ${ }^{1,4}$ ，Tao Liu (刘 涛) ， \\ Shougang Zhang (张首刚) ${ }^{3}$, and Jianyu Wang (王建宇) $)^{1,2}$ \\ ${ }^{1}$ School of Physics and Photoelectric Engineering, Taiji Laboratory for Gravitational Wave Universe, Key Laboratory of Gravitational Wave Precision \\ Measurement of Zhejiang Province, Hangzhou Institute for Advanced Study, University of Chinese Academy of Sciences, Hangzhou 310024, China \\ ${ }^{2}$ Key Laboratory of Space Active Opto-Electronics Technology, Shanghai Institute of Technical Physics, Chinese Academy of Sciences, \\ Shanghai 200083, China \\ 3 Key Laboratory of Time and Frequency Primary Standards, National Time Service Center, Chinese Academy of Sciences, Xi'an 710600, China \\ ${ }^{4}$ School of Physical Sciences, University of Chinese Academy of Sciences, Beijing 100049, China
}

*Corresponding author: jywang@mail.sitp.ac.cn

**Corresponding author: chenlong@ntsc.ac.cn

Received October 14, 2021 | Accepted November 10, 2021 | Posted Online November 25, 2021

\begin{abstract}
A hertz-linewidth ultra-stable laser (USL), which will be used to detect the clock transition line, in a strontium optical clock will be launched into the China Space Station (CSS) in late 2022. As the core of the USL, an interference-filter-based externalcavity diode laser (IF-ECDL) was developed. The IF-ECDL has a compact, stable, and environmentally insensitive design. Performances of the IF-ECDL are presented. The developed IF-ECDL can pass the aerospace environmental tests, indicating that the IF-ECDL can be suitable for space missions in the CSS.
\end{abstract}

Keywords: external-cavity diode laser; interference filter; space application.

DOI: 10.3788/COL202220.021407

\section{Introduction}

The time measurement accuracy of a strontium optical lattice clock (SOLC) has entered the order of $10^{-19}$, which is the highest performance index in the world ${ }^{[1]}$. Due to the microgravity environment of space, performance of the SOLC is expected to be further improved ${ }^{[2,3]}$. In order to achieve this goal, China is developing a space-based SOLC, which will be launched into the China Space Station (CSS) in late 2022. Correspondingly, a hertz-linewidth ultra-stable laser (USL) is also being developed to detect the clock transition line in the SOLC ${ }^{[4]}$. As the light source of the USL ${ }^{[5]}$, the external-cavity diode laser (ECDL) is vital to the whole system.

ECDLs use selective feedback to achieve laser beams with narrow linewidth and tunability ${ }^{[6]}$. The most common ECDL designs are the Littman-Metcalf and the Littrow configurations ${ }^{[7-9]}$. Both of the above-mentioned configurations use a diffraction grating, which is mounted on spring-loaded kinematic structure, as selective feedback. Such designs are sensitive to acoustic, thermal, and mechanical disturbances ${ }^{[10]}$. As a result, the robustness and stability of these ECDLs cannot be guaranteed. Using interference filter (IF) as the selective feedback is another design used to develop an $\mathrm{ECDL}^{[11-14]}$. By adjusting the incidence angle of laser beam on the IF, the longitude mode can be selected. Previous study has proved that the IF-based ECDL (IF-ECDL) is less sensitive to acoustic, thermal, vibration, and mechanical disturbances than LittmanMetcalf or Littrow-based ECDLs ${ }^{[15]}$. With these advantages, the IF-ECDL is currently the most suitable design for space missions among all ECDL designs. Consequently, the IFECDL was selected as the most feasible option in the Project d'Horloge Atomique a Refroidissement d'Atomes en Orbite (PHARO) project, the SOC2 (space optical clock) project in the International Space Station (ISS), and the SOLC in the CSS $^{[16-18]}$. Following that, several prototypes of IF-ECDLs have been developed in laboratories ${ }^{[15,19,20]}$. Performance requirements of the IF-ECDL for the SOLC have been achieved by these prototypes. However, researches on reliability and environmental adaptability of the space-borne IF-ECDLs have not been conducted until now. As a result, there is no literature on the development of an IF-ECDL that can pass the strict aerospace environmental tests. 
In the present work, a compact, high-performance IF-ECDL was developed. The optical and mechanical designs are demonstrated in detail. The IF-ECDL has no moving parts or springloaded structures, which guaranteed high reliability. Titanium and aluminum alloys were used to make structures of the IFECDL. Together with secondary temperature control, the IF-ECDLs have a good thermal design. Performances of the IF-ECDL were measured before and after aerospace environmental tests. Conditions of the aerospace environmental tests are also presented.

\section{Development of the IF-ECDL}

The optical schematic of the developed IF-ECDL is depicted in Fig. 1. The IF-ECDL is based on a cat's eye configuration. An anti-reflection coated laser diode (LD), which has a wavelength of $695-707 \mathrm{~nm}$, is used as the laser source. After emitting from the LD, laser beam is collimated by an aspherical lens (AL1) with a focus length of $4.02 \mathrm{~mm}$. Then, an IF is used to select the longitude modes. By adjusting the laser beam incidence angle to the IF, a $698.445 \pm 0.01 \mathrm{~nm}$ laser is selected to meet the requirement of the SOLC. The laser beam is then focused on a partially reflective mirror (PRM), which has a reflection of $30 \%$ and transmission of $70 \%$, by a cat's eye AL2 with a focus length of $15.29 \mathrm{~mm}$ to form an external cavity. In order to tune the laser frequency, the PRM is glued on a piezoelectric ceramic (PZT) to vary the length of the external cavity. Finally, the laser beam is collimated by AL3 with a focus length of $9.6 \mathrm{~mm}$.

Figure 2 shows the external structure and cross-sectional three-dimensional (3D) view of the IF-ECDL. Considering the vibration and shock during rocket launch, the mechanical strength and reliability of the IF-ECDL should be guaranteed. Other than the protective shell (PS) and heat sink (HS) made

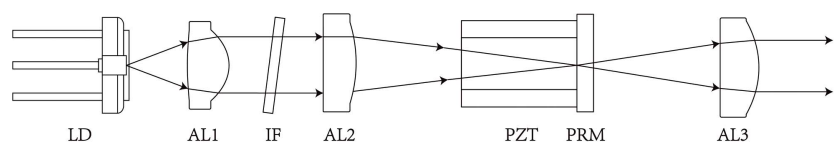

Fig. 1. Optical schematic of the IF-ECDL. LD, laser diode; AL1, aspherical lens 1; IF, interference filter; AL2, aspherical lens 2; PZT, piezoelectric ceramic; PRM, partially reflective mirror; AL3, aspherical lens 3.

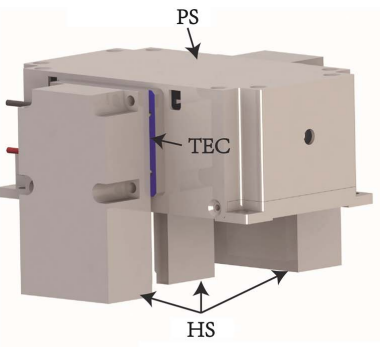

(a)

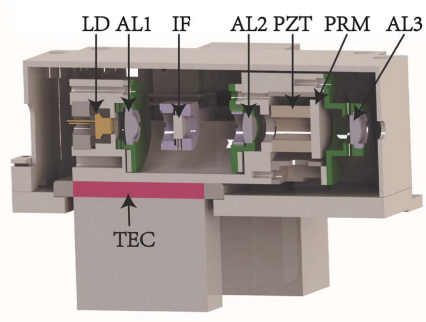

(b)
Fig. 2. IF-ECDL 3D view. (a) External view. (b) Cross-sectional view. PS, protective shell; HS, heat sink; TEC, thermal electric cooler. by aluminum alloy to conduct heat, all other structures in the IF-ECDL are made by titanium alloy. Titanium alloy exhibits high strength and low thermal expansion. The LD is glued into a ring, which is made of aluminum oxide to fix to the main structure. The aluminum oxide ring can maintain insulation while conducting heat. The PRM is firstly glued on the PZT. Then, the PZT is glued on the main structure. The gaps between the PZT and main structure are filled with silicone rubber. Other optical elements in the IF-ECDL are firstly glued on specially designed structures. Then, the structures are fixed to the main structure with screws. Additionally, all screws are glued with epoxy to ensure that the screws will not loosen. The relative distances between different optical elements are designed in OpticStudio (Zemax). Fine adjustments are realized by adding gaskets into structures. When the output power of the laser reaches its maximum, the IF-ECDL can be considered as well adjusted.

While operating in the CSS, the IF-ECDL will be in the experimental cabinet, which contains several payloads. Heat released by other payloads will influence the temperature of the IFECDL, leading to the frequency shifting of laser beam. To avoid this, in addition to the main temperature control of the laser, there is also a secondary temperature control. The main temperature control is realized by a thermal electric cooler (TEC) placed under the LD to precisely control the temperature of the $\mathrm{LD}$. The secondary temperature control is realized by two TECs placed on both sides of the PS of the IF-ECDL separately to keep the temperature of the PS as a constant. Since temperatures of the two temperature control systems cannot be exactly the same, a thermal coupling effect would occur. For weakening this, polyimide gaskets were placed in the middle of the structures of the two temperature systems to isolate heat conduction. With such design, the influence of environmental temperature changes on laser frequency shifting can be weakened. Three HSs are located around the main structure of the IF-ECDL. The HS can connect with the heat conducting board. Thus, heat emitted by the TECs can be taken away by the HS.

With the above-mentioned design, the IF-ECDL was developed. The developed IF-ECDL has a size of $95 \mathrm{~mm} \times 95 \mathrm{~mm} \times$ $55 \mathrm{~mm}$ and a weight of $460 \mathrm{~g}$.

\section{Performances of the IF-ECDL}

Performances of the IF-ECDL were measured. The LD and the TEC used as the primary temperature control of the IF-ECDL were driven by a combined LD and TEC controller (ITC4001, Thorlabs). The TECs used as the secondary temperature control were driven by two separate TEC controllers (TED4015, Thorlabs). The temperature of the IF-ECDL was set as $23.000^{\circ} \mathrm{C}$, while the accuracy of temperature control was less than $0.001^{\circ} \mathrm{C}$.

Figure 3 shows relationships between the electric current and output power of the IF-ECDL and bared LD. From Fig. 3, the threshold of the IF-ECDL is evidently decreased compared to that of the bared LD. The electric current threshold gap is ca. 


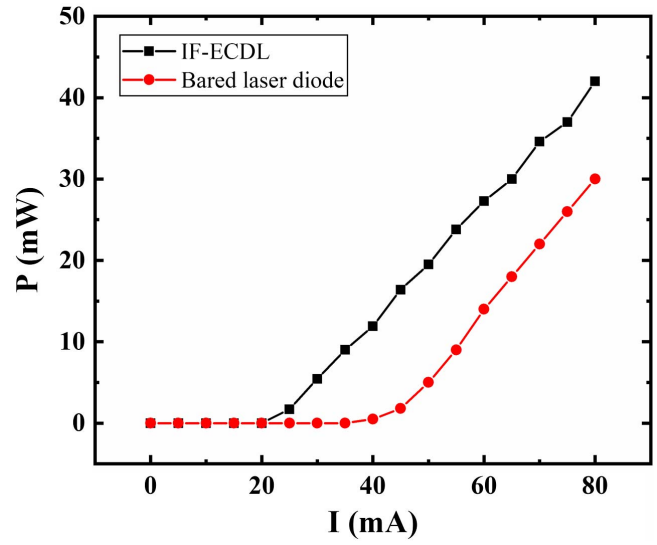

Fig. 3. Electric current versus output power curves of IF-ECDL and bared LD.

$20 \mathrm{~mA}$. This indicates that the external cavity of the IF-ECDL works well, leading to more energy being transferred to laser output power instead of heat. Figure 3 also shows that the IFECDL has a linear current versus power curve after the electric current threshold. Under the circumstance of the electric current being $65 \mathrm{~mA}$ (the current in actual work), the output power of the laser is $30.1 \mathrm{~mW}$, which can meet the requirements of the SOLC.

Figure 4 is the beam quality measurement result of the IFECDL by a beam quality analyzer (Beamsquared, Ophir). The inset in Fig. 4 is the laser spot shape of the IF-ECDL when it works at a current of $65 \mathrm{~mA}$ and temperature of $23.000^{\circ} \mathrm{C}$. Because the LD has a different divergence angle in the horizontal and vertical directions, the shape of the laser spot is an ellipse. Diameters of the laser spot are measured to be 1.67 and $0.89 \mathrm{~mm}$ in the horizontal and vertical directions separately. Figure 4 also shows the beam quality of the IF-ECDL. From the fitted curve, the beam quality factor $M^{2}$ in the horizontal direction is 1.206 , whereas that in the vertical direction is 1.169 . When coupling this laser beam to a polarization maintaining (PM) fiber (PM$630-\mathrm{HP}$ ), the coupling efficiency is higher than $60 \%$ (the value measured in the laboratory). This means that the laser beam can be easily transferred with the PM fiber for mode cleaning or

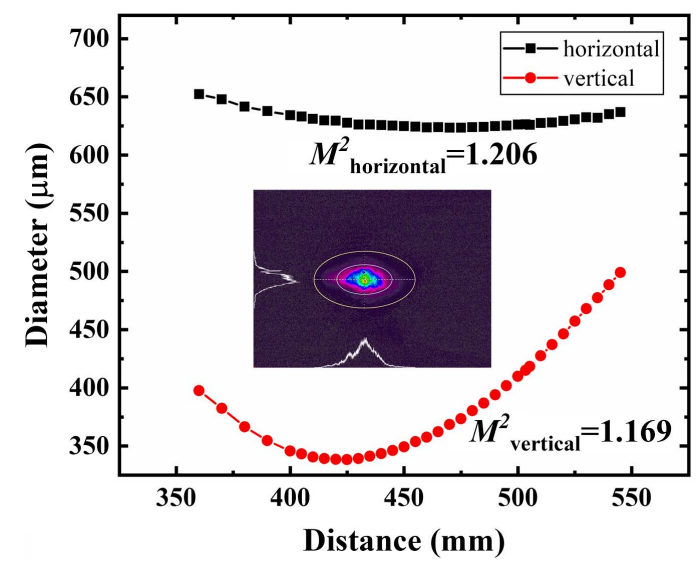

Fig. 4. Beam quality measurement results of the IF-ECDL. connecting with other payloads in the experimental cabinet of the CSS.

The polarization of the laser beam emitted from the IF-ECDL was measured with a polarimeter (PAX1000, Thorlabs). The degree of polarization is $99.83 \%$, meaning that the laser beam can be considered linearly polarized.

To measure the linewidth of the IF-ECDL, an optical heterodyne beam experiment, involving the IF-ECDL and an ultranarrow-linewidth USL system, should be performed. Limited to the circumstance of the lack of an ultra-narrow-linewidth USL system, the linewidth of the IF-ECDL cannot be determined currently. However, based on previous literatures, the linewidth of the IF-ECDL with such a configuration should be at a level of kilohertz $(\mathrm{kHz})^{[15,19,20]}$.

Since the developed IF-ECDL will be used as the light source of the USL in the CSS, the wavelength tuning ability of the IFECDL is vital to the USL. Influences of the PZT, electric current, and temperature on the wavelength tunning of the IF-ECDL were measured with a wavelength meter (WS8-2, Highfinesse). Figure 5 shows the wavelength of the IF-ECDL when adjusting the voltage of the PZT from 0 to $150 \mathrm{~V}$. From Fig. 5, the wavelength of the IF-ECDL can be tuned around a range of $2.5 \mathrm{GHz}$ with the PZT. Figure 5 also demonstrates that the repeatability of the PZT is good. The increasing values of each peak with the increasing voltage can be caused by frequency shift due to temperature change.

Figure 6 shows wavelength of the IF-ECDL when adjusting the $\mathrm{LD}$ electric current. The electric current of the LD varies from 41.1 to $66.0 \mathrm{~mA}$ by adjusting the precision of $0.1 \mathrm{~mA}$. Figure 6(a) shows the overall scan results. From Fig. 6(a), the wavelength tuning range by adjusting the electric current of the $\mathrm{LD}$ is around $45 \mathrm{GHz}$. With the increasing of the $\mathrm{LD}$ electric current, the wavelength of the IF-ECDL decreases in unit of terahertz $(\mathrm{THz})$. When the wavelength of the IF-ECDL decreases to around $429.230 \mathrm{THz}$, it will jump to around $429.275 \mathrm{THz}$. This indicates that the mode-hopping phenomenon occurs. Figure 6(a) also shows that wavelength of the IF-ECDL is not linearly decreased (in units of $\mathrm{THz}$ ) when increasing the electric

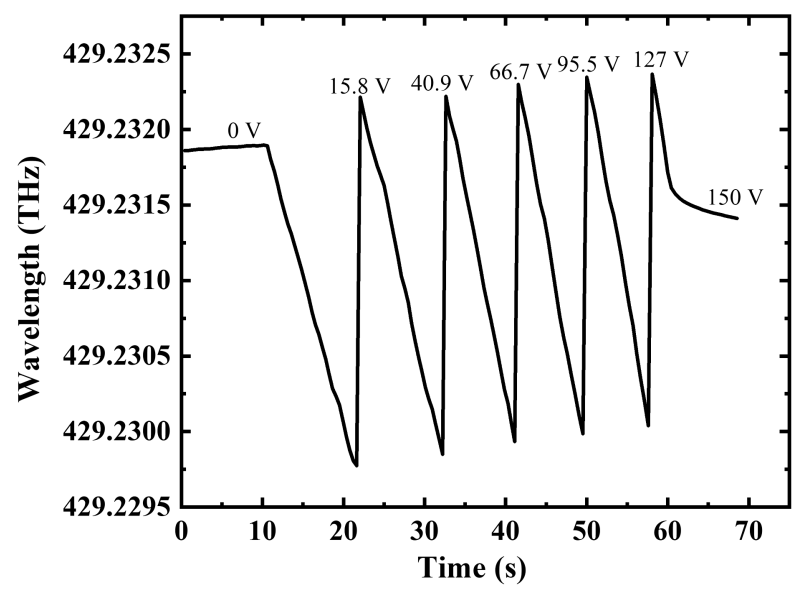

Fig. 5. Wavelength of the IF-ECDL when adjusting the voltage of the PZT. 


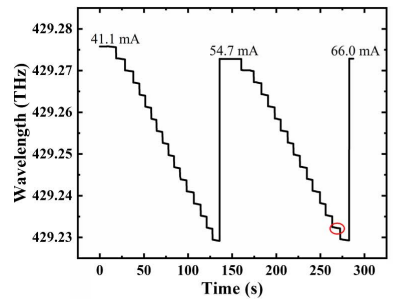

(a)

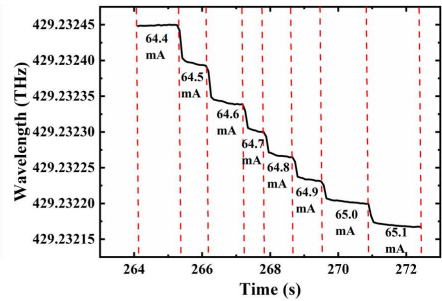

(b)
Fig. 6. Wavelength of the IF-ECDL when adjusting the electric current of the $\mathrm{LD}$ in the range of (a) 41.1 to $66.0 \mathrm{~mA}$ and (b) 64.4 to $65.1 \mathrm{~mA}$.

current linearly. There are steps in the curve. When the electric current of the LD increases, the wavelength of the IF-ECDL will first decrease linearly. Then, after increase of 0.7 or $0.8 \mathrm{~mA}$ of the electric current [as depicted in Fig. 6(b)], the wavelength will suddenly jump and decrease by around $2 \mathrm{GHz}$. This indicates that other than $45 \mathrm{GHz}$ mode-hopping the IF-ECDL also has $2 \mathrm{GHz}$ mode-hopping when adjusting the electric current. Figure 6(b) shows the zoomed data in the red circle of Fig. 6(a). Steps in Fig. 6(b) are caused by the adjustment accuracy of the electric current. In this area, the wavelength of the IF-ECDL can be tuned linearly. The linear tuning range of the IF-ECDL by adjusting the electric current is around $300 \mathrm{MHz}$.

Figure 7 shows the wavelength of the IF-ECDL when adjusting the temperature of the primary temperature control. To avoid the influence of secondary temperature control, only the primary temperature control was on. The results are similar with that caused by the adjustment of the electric current of the IF-ECDL. The phenomenon of wavelength jumps of 45 and $2 \mathrm{GHz}$ also exists. Influences of temperature and electric current on the mode-hopping phenomenon are quite the same. This indicates that mode-hopping is caused by the IF-ECDL itself.

Figure 8 shows the wavelength measurement results of the IFECDL without [indicated in Fig. 8(a)] and with [indicated in Fig. 8(b)] secondary temperature control when operating in the laboratory for $24 \mathrm{~h}$. In Fig. 8(a), under the condition that the secondary temperature control is off, the mode-hopping

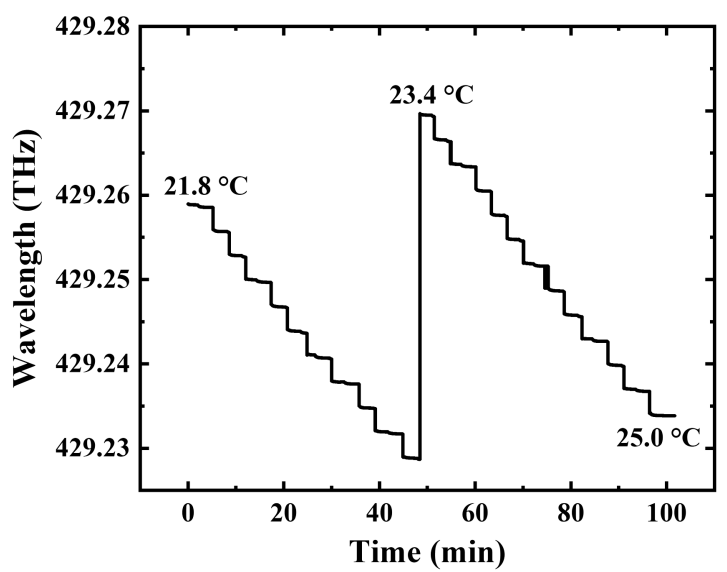

Fig. 7. Wavelength of the IF-ECDL when adjusting the temperature of the LD.

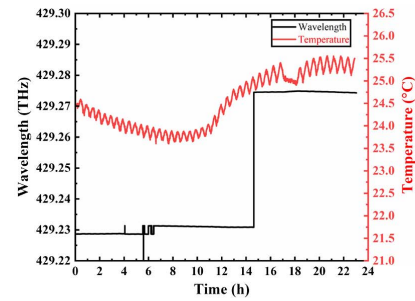

(a)

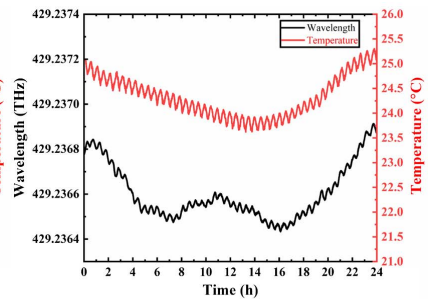

(b)
Fig. 8. Long-term wavelength measurement results of the IF-ECDL under the conditions of (a) without secondary temperature control and (b) with secondary temperature control.

phenomenon occurs. As a result, the wavelength of the IFECDL shifted around $45 \mathrm{GHz}$ in $24 \mathrm{~h}$. Figure 8(b) shows the long-term wavelength of the IF-ECDL when the secondary temperature control is on. From Fig. 8(b), no mode-hopping phenomenon occurred. Although environmental temperature still influences the wavelength of the IF-ECDL, the frequency shift of the IF-ECDL is only around $400 \mathrm{MHz}$, which can be easily compensated by varying the PZT voltage of the IF-ECDL. This indicates that the developed IF-ECDL can be used for long-term operation of the USL when the secondary temperature control is on. From Fig. 8(b), the wavelength of the IFECDL does not agree well with the temperature curve, especially in the time range of $8-16 \mathrm{~h}$. This can be caused by a thermal coupling effect between the main and secondary temperature control systems.

It should be noted that all of above-mentioned laser parameters were measured in a laboratory on Earth. When the IF-ECDL was launched into space, the CSS possessed a microgravity environment. For validating the influence of microgravity on laser performances, the IF-ECDL was measured under the conditions of normal and reverse placement. The changes are within the error range of measurement equipment, indicating that the influence of gravity is negligible.

\section{Aerospace Environmental Tests of the IF-ECDL}

In order to verify that the developed IF-ECDL can be used in the CSS, corresponding aerospace environmental tests were carried out in Hangzhou Saibo Mechanics Environment Test Co., Ltd. Since the IF-ECDL will work inside the CSS, the adaptability of the laser to the vacuum environment is not needed. The IFECDL only needs to pass the environmental tests of mechanical vibrations, shock test, and temperature cycling. Parameters of the tests are based on the requirements of China aerospace standards for payloads inside the CSS. The IF-ECDL for the tests and aerospace environmental tests equipment are shown in Fig. 9.

The vibration tests, including sinusoidal vibrations and random vibrations, of the IF-ECDL were first carried out. According to the schedule, the USL will be launched inside the "Mengtian" laboratory cabin module of the CSS with the Long March 5 rocket. Thus, parameters of the vibration tests are based on the Long March 5 rocket platform, which are 


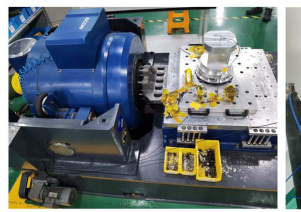

(a)

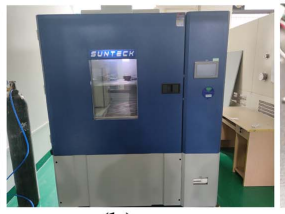

(b)

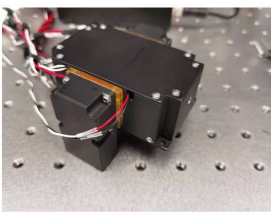

(c)
Fig. 9. Aerospace environmental tests equipment and the IF-ECDL for the tests. (a) Vibration test bench. (b) Temperature test chamber. (c) Developed IF-ECDL for the tests.

shown in Tables 1 and 2. To ensure the reliability of the payloads, all aerospace environmental tests parameters are stricter than actual conditions during rocket launch. With these parameters, the IF-ECDL was tested on three mutually perpendicular axes $(X, Y$, and $Z)$. It should be noted that the unit of amplitude of the sinusoidal vibration test at the frequency of $4-10 \mathrm{~Hz}$ is different than that of other frequencies. This can describe the actual situation of the rocket launch better.

Next, the shock tests of the IF-ECDL were also carried out. The peak acceleration of the shock test is $225 \mathrm{~g}$. The IFECDL was also tested on three mutually perpendicular axes $(X, Y$, and $Z$ ), with three shock tests in each axis.

Finally, the IF-ECDL was put into a temperature test chamber for the temperature cycling test. The highest temperature was set as $50^{\circ} \mathrm{C}$, and the lowest temperature was set as $-10^{\circ} \mathrm{C}$. When the chamber reaches its maximum or minimum temperature, the temperature would hold for $30 \mathrm{~min}$. The changing rate of the temperature was $2^{\circ} \mathrm{C} / \mathrm{min}$. Therefore, the time of one cycle was $90 \mathrm{~min}$. Ten cycles were adopted to test the IF-ECDL.

Table 1. Parameters of the Sinusoidal Vibration Test.

\begin{tabular}{lcc}
\hline Frequency Range $(\mathrm{Hz})$ & Amplitude & Sweep Rate (oct/min) \\
\hline $4-10$ & $9 \mathrm{~mm}$ & 3 \\
$10-17$ & $3.6 \mathrm{~g}$ & 3 \\
$17-16$ & $6.6 \mathrm{~g}$ & 3 \\
$60-100$ & $4.8 \mathrm{~g}$ & 3 \\
\hline
\end{tabular}

Table 2. Parameters of the Random Vibration Test.

\begin{tabular}{lccc}
\hline $\begin{array}{c}\text { Frequency Range } \\
\text { (Hz) }\end{array}$ & $\begin{array}{c}\text { Power Spectral } \\
\text { Density }\end{array}$ & $\begin{array}{c}\text { RMS of Acceleration } \\
\text { (grms) }\end{array}$ & $\begin{array}{c}\text { Time } \\
\text { (s) }\end{array}$ \\
\hline $10-50$ & $\begin{array}{c}\text { dB/oct (rising } \\
\text { slope) }\end{array}$ & 3.055 & 90 \\
$50-300$ & $\begin{array}{c}0.25 \mathrm{~g}^{2} / \mathrm{Hz} \text { (holding } \\
\text { value) }\end{array}$ & 3.055 & 90 \\
$300-2000$ & $\begin{array}{c}\text { (12 dB/oct (falling } \\
\text { slope) }\end{array}$ & 3.055 & 90 \\
& & & \\
\hline
\end{tabular}

Additionally, in order to protect the IF-ECDL from condensation, the chamber was filled with purified nitrogen instead of air.

After each environmental test, the performances of the IFECDL in Section 3 were measured again. The measurement results are basically the same as before the test, which is demonstrated in Section 3. Differences are within the error range of the measurement equipment. Therefore, the developed IFECDL can pass the strict aerospace environmental tests. Consequently, it can be used as the light source of the USL system in the CSS.

\section{Conclusion}

In summary, an IF-ECDL was developed for use in the CSS. The IF-ECDL has a size of $95 \mathrm{~mm} \times 95 \mathrm{~mm} \times 55 \mathrm{~mm}$, a weight of $460 \mathrm{~g}$, a power of $30 \mathrm{~mW}$, and a wavelength of $698.445 \mathrm{~nm}$. The wavelength of the IF-ECDL can be tuned by the LD electric current, temperature, and PZT. The IF-ECDL has primary and secondary temperature control systems. When the accuracy of the temperature control is less than $0.001^{\circ} \mathrm{C}$, the IF-ECDL can work $24 \mathrm{~h}$ without the mode-hopping phenomenon occurring. Due to the compact, stable, and environmentally insensitive design, the IF-ECDL can pass the aerospace environmental tests. Therefore, it can be used as the laser source of the USL in the CSS.

\section{Acknowledgement}

This work was supported by the National Key R\&D Program of China (No. 2020YFC2201300) and the National Natural Science Foundation of China (No. 11903041).

\section{References}

1. S. L. Campbell, R. B. Hutson, G. E. Marti, A. Goban, N. D. Oppong, R. L. Mcnally, L. Sonderhouse, J. M. Robinson, W. Zhang, B. J. Bloom, and J. Ye, "A Fermi-degenerate three-dimensional optical lattice clock," Science 358, 90 (2017).

2. S. Origlia, S. Schiller, M. S. Pramod, L. Smith, Y. Singh, W. He, S. Viswam, D. Swierad, J. Hughes, K. Bongs, U. Sterr, Ch Lisdat, S. Vogt, S. Bize, J. Lodewyck, R. Le Targat, D. Holleville, B. Venon, P. Gill, G. Barwood, I. R. Hill, Y. Ovchinnikov, A. Kulosa, W. Ertmer, E. M. Rasel, S. Stuhler, and W. Kaenders, "Development of a strontium optical lattice clock for the SOC mission on the ISS," Proc. SPIE 9900, 990003 (2016).

3. S. Origlia, M. S. Pramod, S. Schiller, Y. Singh, K. Bongs, R. Schwarz, A. AlMasoudi, S. Dörscher, S. Herbers, S. Häfner, U. Sterr, and C. Lisdat, "Towards an optical clock for space: compact, high-performance optical lattice clock based on bosonic atoms," Phys. Rev. A 98, 053443 (2018).

4. Y. Sun, Y. Yao, Y. Hao, H. Yu, Y. Jiang, and L. Ma, "Laser stabilizing to ytterbium clock transition with Rabi and Ramsey spectroscopy," Chin. Opt. Lett. 18, 070201 (2020).

5. X. Chen, Y. Jiang, B. Li, H. Yu, H. Jiang, T. Wang, Y. Yao, and L. Ma, "Laser frequency instability of $6 \times 10^{-16}$ using $10-\mathrm{cm}$-long cavities on a cubic spacer," Chin. Opt. Lett. 18, 030201 (2020).

6. K. C. Harvey and C. J. Myatt, "External-cavity diode laser using a grazingincidence diffraction grating," Opt. Lett. 16, 910 (1991).

7. L. Ricci, M. Weidemüller, T. Esslinger, A. Hemmerich, C. Zimmermann, V. Vuletic, W. König, and T. W. Hänsch, “A compact grating-stabilized diode laser system for atomic physics," Opt. Commun. 117, 541 (1995).

8. A. S. Arnold, J. S. Wilson, and M. G. Boshier, "A simple extended-cavity diode laser,” Rev. Sci. Instrum. 69, 1236 (1998). 
9. B. Mroziewicz, "External cavity wavelength tunable semiconductor lasers - a review," Opto-Electron. Rev. 16, 347 (2008).

10. D. J. Thompson and R. E. Scholten, "Narrow linewidth tunable external cavity diode laser using wide bandwidth filter,” Rev. Sci. Instrum. 83, 023107 (2012).

11. X. Baillard, A. Gauguet, S. Bize, P. Lemonde, P. Laurent, A. Clairon, and P. Rosenbusch, "Interference-filter-stabilized external-cavity diode lasers," Opt. Commun. 266, 609 (2016).

12. P. Zorabedian and W. R. Trutna, "Interference-filter-tuned, alignmentstabilized, semiconductor external-cavity laser," Opt. Lett. 13, 826 (2012).

13. Z. Jiang, Q. Zhou, Z. Tao, X. Zhang, S. Zhang, C. Zhu, P. Lin, and J. Chen, "Diode laser using narrow bandwidth interference filter at $852 \mathrm{~nm}$ and its application in Faraday anomalous dispersion optical filter," Chin. Phys. B 25, 083201 (2016).

14. C. Wei, C. Zuo, L. Liang, and S. Yan, "Compact external cavity diode laser for quantum experiments,” Optoelectron. Lett. 16, 433 (2020).

15. L. Zhang, T. Liu, L. Chen, G. Xu, C. Jiang, J. Liu, and S. Zhang, "Development of an interference filter-stabilized external-cavity diode laser for space applications," Photonics 7, 12 (2020)
16. E. Simon, C. Coatantiec, M. Saccoccio, D. Blonde, J. Loesel, P. Laurent, I. Maksimovic, and M. Abgrall, "A highly stable frequency stabilized extended cavity diode laser for space," Proc. SPIE 5249, 203 (2004).

17. T. Lévèque, B. Faure, F. X. Esnault, C. Delaroche, D. Massonnet, O. Grosjean, F. Buffe, P. Torresi, T. Bomber, A. Pichon, P. Béraud, J. P. Lelay, S. Thomin, and Ph. Laurent, "PHARAO laser source flight model: design and performances," Rev. Sci. Instrum. 86, 033104 (2015).

18. D. Świerad, S. Häfner, S. Vogt, B. Venon, D. Holleville, S. Bize, A. Kulosa, S. Bode, Y. Singh, K. Bongs, E. M. Rasel, J. Lodewyck, R. L. Targat, C. Lisdat, and U. Sterr, "Ultra-stable clock laser system development towards space applications,” Sci. Rep. 6, 33973 (2016).

19. J. Ruan, J. Liu, J. Ma, Z. Du, C. Wu, and S. Zhang, "Robust external cavity diode laser system with high frequency stability for Cs atomic clock," Chin. Opt. Lett. 8, 300 (2010).

20. A. Wicht, A. Bawamia, M Krüger, Ch Kürbis, M. Schiemangk, R. Smol, A. Peters, and G. Tränkle, "Narrow linewidth diode laser modules for quantum optical sensor applications in the field and in space," Proc. SPIE 10085, 100850F (2017). 